\title{
Anabases
}

ANABASES Traditions et réceptions de l'Antiquité

$24 \mid 2016$

Varia

\section{La femme étrusque et son époux à travers l'épigraphie}

\section{Lavinia Magnani}

\section{OpenEdition}

Journals

Édition électronique

URL : http://journals.openedition.org/anabases/5874

DOI : 10.4000/anabases.5874

ISSN : 2256-9421

\section{Éditeur}

E.R.A.S.M.E.

\section{Édition imprimée}

Date de publication : 10 novembre 2016

Pagination : 63-75

ISSN : 1774-4296

\section{Référence électronique}

Lavinia Magnani, «La femme étrusque et son époux à travers l'épigraphie », Anabases [En ligne], 24

2016, mis en ligne le 01 octobre 2019, consulté le 24 mars 2020. URL : http://

journals.openedition.org/anabases/5874 ; DOI : https://doi.org/10.4000/anabases.5874 


\section{La femme étrusque et son époux à travers l'épigraphie}

Lavinia Magnani

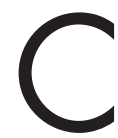
ette étude a pour objet le rôle social et familial que remplissait la femme étrusque en tant qu'épouse. J'aborderai la question à travers l'épigraphie, et plus spécifiquement à travers l'analyse du matronyme, un élément propre à l'onomastique étrusque et qui en fait l'originalité.

Les Étrusques de naissance libre utilisaient un nom composite presque équivalent à celui des Romains, avec trois éléments principaux, le praenomen (nom individuel), le nomen (nom familial) et le patronyme (le nom du père), éventuellement accompagnés d'un ou plusieurs cognomina (surnoms). Mais la formule onomastique étrusque complète comprenait aussi un matronyme, le nom de la mère.

Exemple de nom romain : Caius Cornelius Caïfilius

Caius est le praenomen, Cornelius le nomen, Caïfilius, signifiant « fils de Caius », est le patronyme.

Exemple de nom étrusque : Vel Partunus Velus clan Ramthas Cuclnial

Vel est le praenomen, Partunus le nomen, Velus clan, signifiant « fils de Vel », est le patronyme, Ramthas Cuclnial, signifiant « de Ramtha Cuclni », est le matronyme.

Cette particularité de l'onomastique étrusque est souvent évoquée lorsqu'il s'agit de discuter la condition des femmes en Étrurie, généralement considérée comme meilleure que celles des femmes des sociétés méditerranéennes de la même époque que l'on connaît, notamment les Grecs et les Romains. C'est une idée ancienne qui a été reprise par les études modernes et qui se fonde sur plusieurs éléments, en premier lieu sur les commentaires des auteurs grécolatins. Selon Théopompe ${ }^{1}$ les femmes étrusques jouissaient d'une liberté sexuelle

1 apud Atheneum, XII, 5ı7d. 
inconcevable pour une matrone romaine: elles participaient aux banquets, s'étendaient sur la kliné avec le premier venu au lieu de rester discrètement assises (une accusation vaguement reprise par Aristote, selon qui les Étrusques soupaient avec leur femme, couchés sous la même couverture ${ }^{2}$ ), buvaient en quantité, faisaient libre commerce de leur corps et s'exerçaient nues même en présence d'hommes. En outre, Timée ${ }^{3}$ affirme que les esclaves étrusques servaient les convives sans être vêtus et dans la Cistellaria de Plaute, l'esclave Lampadion fait référence à la coutume répandue parmi les femmes étrsuques de se procurer une dot à travers la prostitution ${ }^{4}$. L'image de débauche que nous peignent les auteurs gréco-latins n'est confirmée ni par l'archéologie ni par l'iconographie. Ces propos relèvent pour la plupart de la médisance et de la calomnie fantaisiste, mais ils suggèrent que les femmes étrusques avaient des droits ou des comportements susceptibles d'être mal compris par des sociétés plus patriarcales.

D'autre part, l'historiographie romaine nous a transmis le souvenir de plusieurs femmes d'origine étrusque qui se sont distinguées par leur tempérament énergique et leur influence sur la vie politique : Tanaquil l'épouse de Tarquin l'Ancien, Tullia Minor l'épouse de Tarquin le Superbe, et l'autoritaire aristocrate Urgulania, qui, en raison de son amitié avec l'impératrice Livie, se croyait au-dessus de la loi. Que ces figures aient réellement existé ou qu'elles relèvent d'une construction imaginaire, elles sont révélatrices des caractéristiques que la mentalité classique attribuait aux femmes étrusques et, au moins en ce qui concerne la participation des femmes à la vie sociale, les sources littéraires sont confirmées par l'archéologie.

L’iconographie funéraire nous montre des épouses étrusques étendues sur la kliné avec leurs maris, et d'autres petits indices renforcent l'image d'une femme étrusque participant aux manifestations collectives de sa cité: sur les parois de la Tomba delle Bighe, par exemple, sont peints des jeux publics dont les spectateurs appartiennent aux deux sexes.

Mais une place d'honneur dans la discussion scientifique sur la condition féminine en Étrurie revient à l'onomastique, où deux aspects sont régulièrement évoqués. Premièrement, la femme étrusque avait un nom bi-membre tout à fait équivalent à celui des hommes : praenomen + nomen, alors que la femme romaine ne possédait pas de praenomen. Officiellement, elle était connue par le seul gentilice, une désignation collective de l'ensemble des membres de la famille, ce qui lui niait donc une identité individuelle. Deuxièmement, la formule onomastique étrusque, aussi bien masculine que féminine, pouvait comprendre le matronyme,

2 apud Atheneum, I, $23 \mathrm{~d}$.

3 apud Atheneum, IV, I53 d.

4 Plaute, Cistellaria, II, 3, 562-3. 
ce qui suggère que la matrone étrusque, loin d'être seulement un moyen pour l'homme d'avoir une descendance, était sur un pied d'égalité avec son mari.

Remarqués depuis longtemps, ces éléments ont suscité des interprétations parfois très audacieuses de la condition féminine en Étrurie. Dans sa Die Sage von Tanaquil (1870), J. J. Bachofen a défini la société étrusque comme un exemple de Mutterrecht (matriarcat) survivant à l'époque historique. Les interprétations successives ont été plus prudentes. En ig6r, J. Heurgon a rectifié l'expression de J. J. Bachofen: ce n'est pas de Mutterrecht qu'il faut parler, mais de Mutterkultur ${ }^{5}$. Selon J. Heurgon, il est invraisemblable et injustifié de supposer que les femmes étrusques, comme un véritable matriarcat le prévoit, détenaient le pouvoir institutionnel, économique, social et religieux ${ }^{6}$. J. Heurgon, néanmoins, affirme que la société étrusque était marquée par des modèles sociaux et culturels d'origine non-patriarcale, où les femmes n'étaient pas souveraines mais avaient souvent le dernier mot.

L'association entre le peuple étrusque et cette forme de féminisme ante litteram est désormais répandue dans la littérature ${ }^{7}$. Cependant, il nous semble que toutes les facettes du sujet n'ont pas été traitées de façon véritablement scientifique. Le matronyme, en particulier, est un élément constamment présent dans les études, mais dont le caractère problématique a été quelque peu négligé. Le fait que le matronyme existe, à lui seul, n'est pas un signe incontestable du prestige de la femme étrusque, de son poids en tant qu'individu, de son autorité à l'intérieur de la famille. En effet, le comportement épigraphique étrusque est plus variable que son équivalent romain, et le choix des éléments onomastiques inconstant. Il faut déterminer exactement les conditions d'utilisation du matronyme pour pouvoir comprendre la valeur de cet élément onomastique et en tirer des informations sur le rôle que remplissait la matrone dans la société étrusque. L'interprétation traditionnelle du matronyme a été remise en question par $\mathrm{E}$. Benelli ${ }^{8}$ : dans un article de 200I, il remarque que le matronyme apparaît presque exclusivement dans des

5 J. Heurgon, “Valeurs féminines et masculines dans la civilisation étrusque », Mélanges de l'École française de Rome 73 (196), p. 139-16o.

6 Pour une définition de matriarcat: C. G. Thомas, « Matriarchy in Early Greece : Bronze and Dark Ages », Arethusa 6, 2 (1973), p. i73 et ı8o.

7 Voir, entre autres : L. Bonfante, “ The Women of Etruria », Arethusa 6, I (I973), p. 9I-IoI ; M. Sordi, "La donna etrusca ", in Misoginia e maschilismo in Grecia e in Roma, Gênes, I98I, p. 49-67; A. Rallo (a cura di), Le donne in Etruria, Rome, I989; le plus récent P. Amann, Die Etruskerin. Geschlechterverhältnis und Stellung der Frau im frühen Etrurien, Vienne, 2000, fait le point sur la discussion.

8 E. BenELLI, « Le formule onomastiche della Tabula Cortonensis e il valore del metronimico ", in La Tabula Cortonensis e il suo contesto storico-archeologico. Atti dell'Incontro di Studio, Roma 200I, Rome, 2002, p. 93-Ioo. 
contextes funéraires privés et cachés, alors que les inscriptions sur support extérieur présentent une formule onomastique plutôt simplifiée et régulière qui exclut le matronyme. Cela amène E. Benelli à postuler l'existence d'une formule onomastique officielle qui comprend, outre praenomen et nomen, le patronyme seul. D'autre part, il remarque que l'emploi du matronyme dans la Table de Cortone est réservé aux personnes susceptibles d'être confondues avec d'autres en raison de phénomènes d'homonymie et peut ainsi s'expliquer par la simple nécessité de distinguer les individus. En montrant ainsi le caractère problématique de l'emploi du matronyme, E. Benelli a ouvert une piste de recherche qui mérite d'être poursuivie.

La présente discussion se fonde sur une expérience méthodologique ${ }^{9}$ dont le but est d'analyser la signification du matronyme à partir de son contexte d'utilisation. Nous avons pris comme échantillon 500 inscriptions onomastiques d'époque récente constituant le corpus épigraphique de Tarquinia ${ }^{10}$, provenant à la fois de sarcophages, parois et cippes. Nous les avons étudiées suivant deux types de démarches : premièrement, une approche statistique du corpus permettant de découvrir les critères généraux d'utilisation du matronyme. Deuxièmement, l'étude détaillée d'ensembles funéraires où apparaissent des matronymes, pour comprendre quelles fonctions remplit cet élément onomastique dans un contexte très spécifique. Nous présenterons une de ces études de cas dans la deuxième partie de ce texte.

9 Cette discussion représente un compte-rendu du travail effectué pour mon Mémoire de master : L. Magnani, "Le matronyme étrusque et ses implications socio-culturelles : analyse du corpus épigraphique de Tarquinia", Mémoire de master en Lettres Classiques, sous la direction de Dominique Briquel, Paris, Université Paris-Sorbonne, 2013.

10 Tel qu'il est présenté par F. Chiesa, Tarquinia : archeologia e prosopografia tra ellenismo e romanizzazione, Rome, 2005. Le corpus est numériquement important mais ne présente que les inscriptions suffisamment bien connues et documentées. Centre majeur de la culture étrusque, Tarquinia est un champ de recherche riche : aux hypogées souterrains s'ajoute une copieuse production de cippes, ce qui nous permettra d'examiner le bien-fondé de la théorie d'E. Benelli, selon laquelle les matronymes appartiennent uniquement aux inscriptions sur support interne. Nous nous limiterons aux inscriptions d'époque récente puisque l'écriture n'apparaît qu'occasionnellement dans la phase archaïque. 


\section{Observations générales sur l'emploi du matronyme à Tarquinia}

D’un regard général sur les 5oo inscriptions onomastiques de Tarquinia, on peut tirer les informations suivantes.

Fig. I et fig. 2 : sur la totalité des inscriptions, I7\% présentent un matronyme, $4 \mathrm{I} \%$ présentent un patronyme. On en déduit que le matronyme fait partie de la culture étrusque de Tarquinia mais ce n'est pas un élément ordinaire et régulier. Le patronyme est beaucoup plus présent.

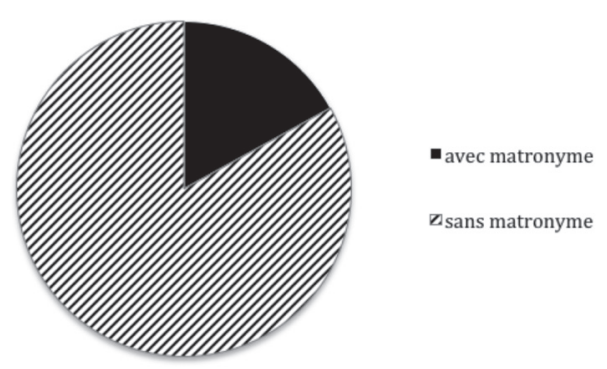

Fig. I. Proportion de matronymes sur la totalité des inscriptions

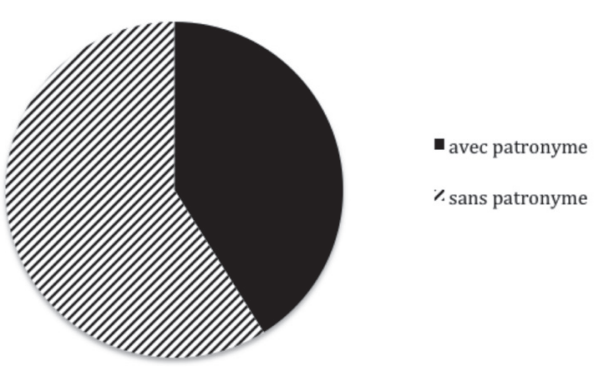

Fig. 2. Proportion de patronymes sur la totalité des inscriptions

Fig. 3 : les inscriptions présentant un matronyme seul (et pas de patronyme) constituent 2\% du total des inscriptions. On en déduit que non seulement le matronyme est moins répandu que le patronyme mais il semble aussi être dépendant du patronyme. Il peut difficilement apparaître seul. C'est un élément onomastique subordonné, ce qui suggère que, d'un point de vue onomastique, père et mère ne sont pas équivalents.

Fig. 4 et fig. 5 : 6\% des matronymes apparaissent sur support externe (sur les cippes), contre $25 \%$ des patronymes. Il est donc clair que le contexte privilégie du matronyme est l'hypogée souterrain et non-visible.

Fig. 6 : le patronyme est habituellement constitué par le praenomen du père. Le matronyme constitué par le seul praenomen de la mère est un cas de figure virtuellement inexistant. $45 \%$ des matronymes de Tarquinia se composent du praenomen + nomen de la mère, $55 \%$ par le nomen seul. Ce constat tout simple est la clé de voûte de la discussion. Si on désignait si souvent la mère uniquement par son nom de famille, il nous semble flagrant que la priorité n'allait pas à l'individualité de la mère mais plutôt à ses origines familiales.

Cette vue d'ensemble confirme en grande partie les observations d'E. Benelli: les matronymes sur support extérieur sont rares. Le contexte privilégié du matronyme est l'hypogée, c'est-à-dire la tombe familiale souterraine. À titre d'exemple, nous nous pencherons sur l'un de ces hypogées, la Tombe des Boucliers, où sont 
bien résumées les différentes pistes de réflexion qu'on peut suivre pour interpréter le matronyme. En outre, la Tombe des Boucliers présente l'avantage d'être très riche en informations prosopographiques, ce qui facilite beaucoup un travail sur les dynamiques familiales étrusques et la place qu'elles laissaient à la femme.

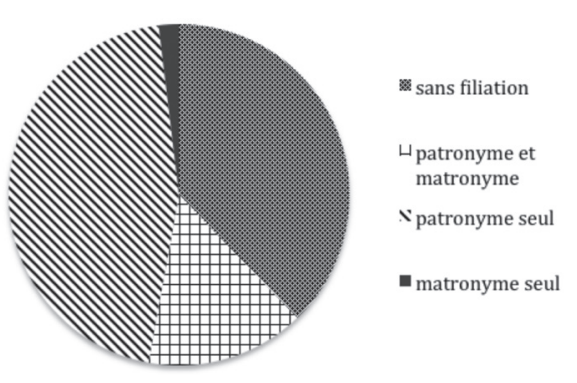

Fig. 3. Répartition des matronymes et patronymes sur les inscriptions totales

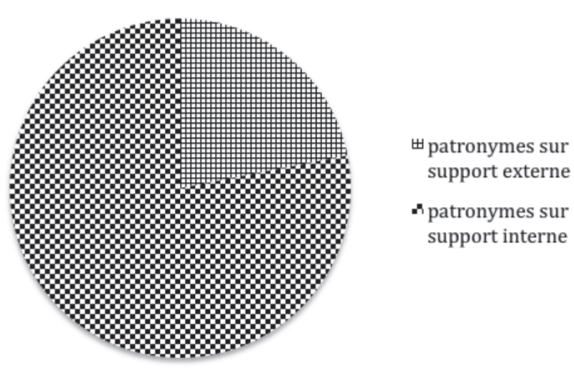

Fig. 5. Proportion de patronymes sur support externe et interne

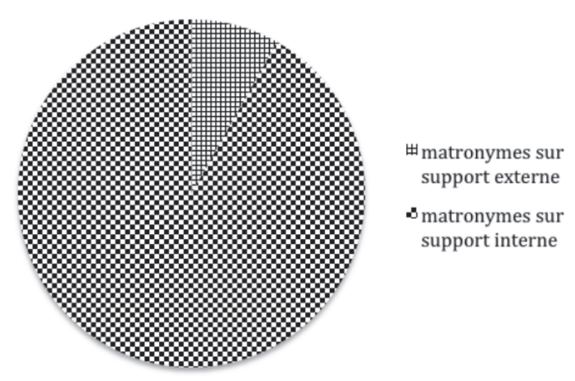

Fig. 4. Proportion de matronymes sur support externe et interne

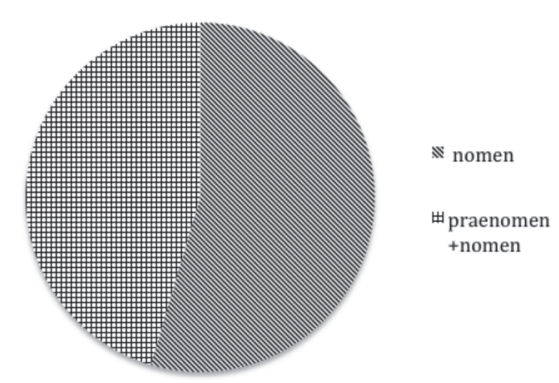

Fig. 6. Forme du matronyme

\section{La Tombe des Boucliers}

La Tombe des Boucliers est un hypogée de la fin du IV siècle, situé dans la nécropole de Monterozzi. Le $C I E$ propose une généalogie de la famille Velcha reconstruite à partir des inscriptions onomastiques de l'hypogée (fig. 7). La tombe est composée de quatre chambres, indiquées par les lettres A, B, C et D (fig. 8). Les chambres $B$, C et $D$ étaient des espaces véritablement funéraires, $B$ étant le principal, avec quatorze boucliers peints sur ses parois pour représenter sans doute les quatorze personnes qui y étaient déposées. La chambre A avait une fonction décorative et commémorative. Elle est entièrement décorée par une fresque à figures humaines. Les Velcha y apparaissent, identifiés presque tous par des didascalies. 


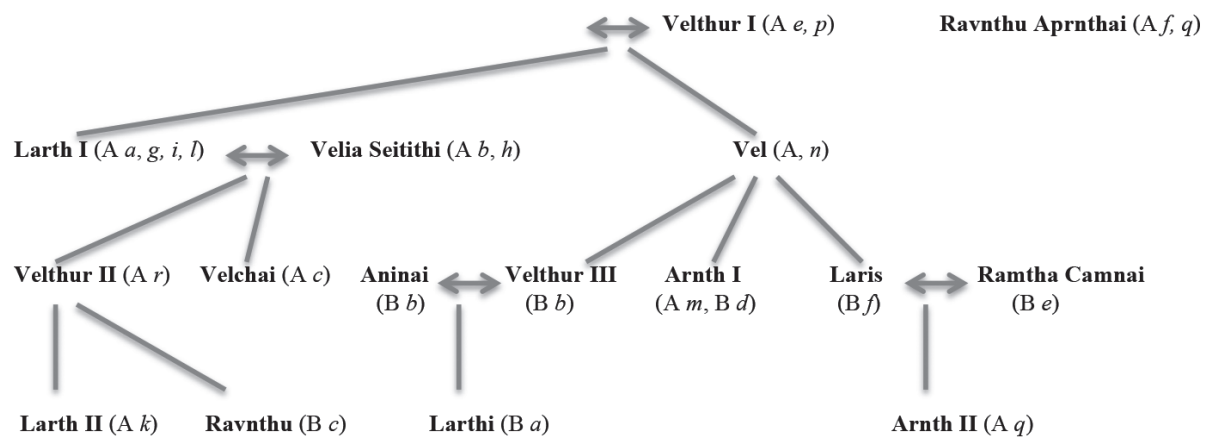

Fig. 7. Généalogie des Velcha établie par O. A. Danielsson dans le CIE II, I, 3. Le schéma a été reproduit par l'auteur, avec l'ajout des références aux épitaphes relatives à chaque membre de la famille (A $q=$ chambre A, épitaphe $q$ )

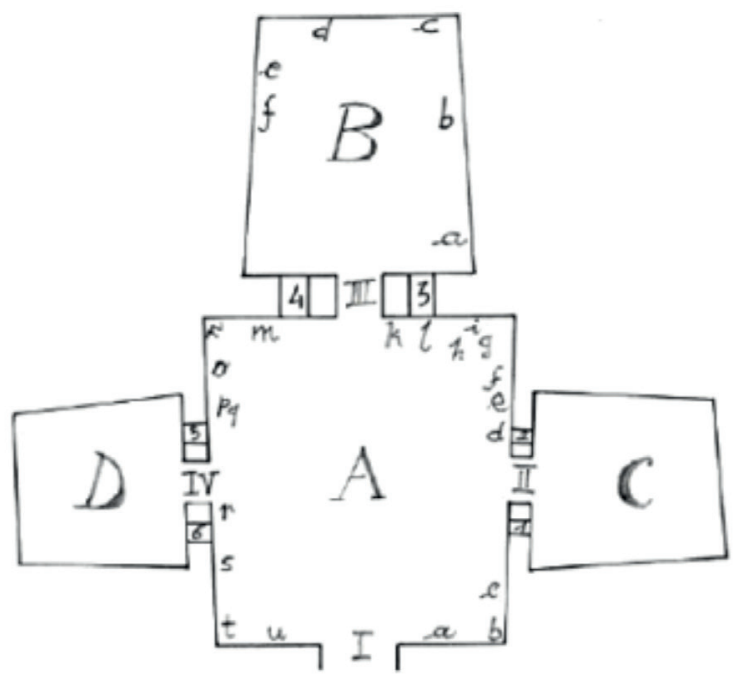

Fig. 8. Plan de la Tombe des Boucliers.

Les petites lettres correspondent aux inscriptions pariétales

Nous reconnaissons trois grandes scènes ${ }^{11}$ : un cortège de magistrats guidé par Larth I, fondateur de l'hypogée, auquel participent d'autres membres de la famille ; une scène de banquet dans l'Au-delà, avec, d'un côté, Larth I et sa femme Velia

11 C'est l'interprétation traditionnelle : voir M. Torelli, L'arte degli Etruschi, Milan, I985, p. 222-23o. 
Seitithi, de l'autre côté, Velthur I et Ravnthu Aprthnai, parents de Larth I ; l'héroïsation de Velthur I, avec sa femme Ravnthu Aprthnai comme spectatrice. Nous trouvons vingt inscriptions pariétales dans la chambre A, à la fois des didascalies identifiant les personnages peints et des épitaphes indiquant qui est déposé dans les chambres adjacentes. Six autres épitaphes pariétales se trouvent dans la chambre B et une dans la chambre C.

Aussi bien la décoration que la disposition des sépultures dans cette tombe mettent en valeur le couple et les noyaux familiaux : le fondateur Larth I et sa femme Velia Seitithi sont représentés ensemble en train de banqueter, ainsi que Velthur I et sa femme Ravnthu Aprthnai, représentés une deuxième fois lors de l'héroïsation de Velthur I. Dans la scène du cortège, Larth I, Velia Seitithi et leurs enfants Velthur II et Velchai apparaissent ensemble, ainsi que leurs cousins, Velthur III avec sa femme Aninai et leur fille Larthi. Ces trois dernières personnes sont déposées côte à côte dans la chambre B. De même, le frère de Velthur III, Laris, est déposé avec sa femme.

Les matronymes, en revanche, ne sont pas très fréquents. Même si la tombe est très riche en inscriptions onomastiques, il n'y a que trois matronymes, contre onze patronymes. Cela nous confirme encore une fois que le matronyme est un élément plutôt rare en épigraphie. Deux matronymes font partie de deux longues inscriptions dédiées au fondateur Larth I : la première (A $i$ ) se trouve au-dessous du personnage de Larth I en train de banqueter. C'est une longue inscription dont la lecture est beaucoup discutée.

Lecture de H. Rix ${ }^{12}$ :

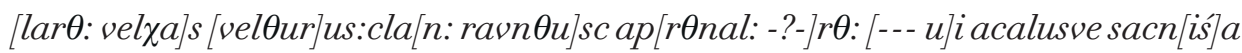

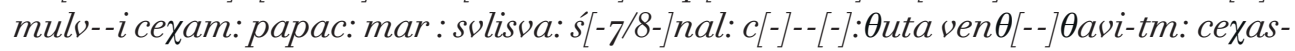

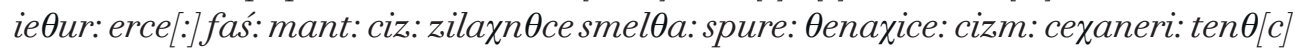

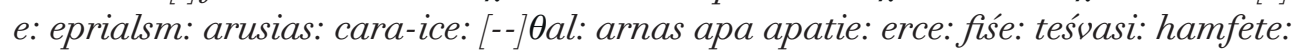

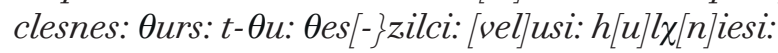

Le début de l'inscription peut facilement être reconstruit et traduit : [lar $\theta$ :

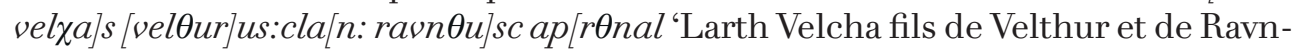
thu Aprthnai'. Sans lire le reste du texte dans son intégralité, nous pouvons certainement remarquer deux références à des charges politiques ${ }^{13}$ : Larth a été trois

12 H. Rix, ET, Ta 5.4 (CIE 5385).

13 Pour la lecture de cette inscription, voir aussi A. Morand, “ La Tomba degli Scudi di Tarquinia: contributo epigrafico per l'esegesi dei soggetti », Mélanges de l'École française de Rome 99, 1987, p. 95-ıाо. 


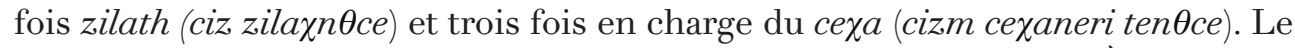
zilacat étant la plus haute magistrature du cursus honorum étrusque ${ }^{14}$. À l'intérieur $\mathrm{du}$ zilacat, on distingue plusieurs grades ou fonctions, dont le cex $a^{15}$. L'inscription dans son ensemble paraît être une forme d'elogium pour Larth I et sa brillante carrière politique.

La deuxième inscription avec matronyme (A l) n'est ni une didascalie ni une épitaphe : elle commémore la fondation de l'hypogée.

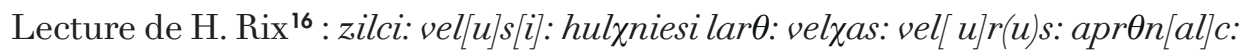

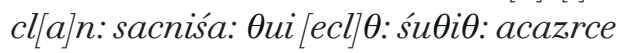

On peut donner de cette inscription une traduction relativement sûre : 'Sous la magistrature de Vel Huchnie, Larth Velchas, fils de Velthur et de l'Aprthnai, a consacré cette tombe ${ }^{17}$. Vel Huchnie est le magistrat éponyme qui apparaît aussi dans l'elogium de Larth I. Cette inscription confirme que Larth I est le fondateur de la tombe, ce qui est cohérent avec la présence de l'elogium et la place importante accordée au personnage dans le programme décoratif.

Le troisième matronyme (A $r$ ) apparaît dans une épitaphe peinte sur le mur de la chambre $\mathrm{A}$ adjacent à la chambre $\mathrm{D}$, pour indiquer que Velthur y est déposé.

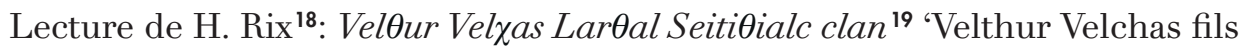
de Larth et de la Seitithi'.

Nous pouvons remarquer que deux des trois inscriptions avec matronyme (A $i$ et $\mathrm{A} l$ ) partagent une dimension quelque peu solennelle : elles ont pour but de perpétuer la mémoire d'un personnage illustre et d'un événement important dans

14 Il est souvent mentionné deux fois, au début et à la fin de la liste des charges politiques, ou tout seul, comme résumé implicite de toute une carrière: A. MagGiani, "Appunti sulle magistrature etrusche », Studi Etruschi 62, ı998, p. ı26.

15 Voir M. Pallottino, Etruscologia, Milan, I984, p. 507; A. Maggiani, “Appunti sulle magistrature etrusche », Studi Etruschi 62, I998, p. I27.

16 H. Rix, ET, Ta 5.5 (CIE 5388).

17 G. Facchetri, Appunti di morfologia etrusca. Con un'Appendice sulla questione delle affinità genetiche dell'etrusco, Florence, 2002. p. 59, note 2I.

18 H. Rix, ET, Ta ı.56 (CIE 5395).

19 Les inscriptions dont la lecture et l'interprétation ne posent pas de difficultés sont, par commodité, transcrites sans signes diacritiques, avec des majuscules pour les noms propres. 
l'histoire familiale. Cela reflète une tendance générale à Tarquinia d'insérer les matronymes dans des inscriptions hors du commun, longues, élaborées, donnant plus d'informations que la simple formule onomastique du défunt ${ }^{20}$. Remarquons aussi que deux matronymes sur trois $(\mathrm{A} l, \mathrm{~A} r)$ omettent le praenomen de la mère. Nous avons déjà souligné que, dans le corpus épigraphique de Tarquinia, le matronyme met beaucoup plus l'accent sur le nomen maternel que sur son praenomen. Il peut être donc profitable de se pencher sur l'origine familiale des femmes en question.

Dans le cas de la Tombe des Boucliers, il s'agit des gentes Seitithe et Aprthna. Les Aprthna sont malheureusement très peu documentés et leurs seules relations connues sont celles qu'ils ont entretenues avec les Velcha. Le gentilice Aprthna est dérivé du nom individuel Apurthe avec ajout du suffixe d'appartenance - $n a^{21}$. Cette origine patronymique du gentilice suggère qu'on a affaire à une famille ancienne, mais pour le reste, il vaut mieux ne pas tenter de reconstruire une histoire dont on ne peut rien savoir avec certitude. Les Seitithe, de leur côté, pourraient avoir des origines plus récentes. En effet, les gentilices à suffixe - at/ $\theta e$ dérivent régulièrement d'adjectifs ethniques que l'on imagine avoir été attribués à des étrangers graduellement intégrés dans le tissu social ${ }^{22}$. Mais même si les Seitithe ne peuvent pas être comptés parmi les vieilles familles de l'aristocratie de Tarquinia, ils entretenaient avec elles de très bons rapports. Des Seitithi ont été accueillies en mariage par des familles distinguées : les Velcha, les Apuna, les Phursethna ${ }^{23}$. Il est très intéressant de remarquer que la famille Seitithe n'est connue que par ses représentantes féminines, dont les noms apparaissent dans les hypogées de leurs maris. Une Seitithi a épousé un Apuna de la Tombe Bruschi. On retrouve aussi le gentilice à Chiusi et à Vulci (toujours par des représentantes féminines). Il semblerait donc que les Seitithe s'étaient résolument engagés dans une politique d'alliances matrimoniales.

C'est précisément ce système particulier de relations sociales qui pourrait avoir influencé l'emploi du matronyme. Si on réunit les différents éléments discutés jusqu'ici (la mise en valeur de l'épouse dans l'iconographie et la disposition

20 D’autres exemples sont analysés dans mon Mémoire : L. Magnani, "Le matronyme étrusque et ses implications socio-culturelles : analyse du corpus épigraphique de Tarquinia", Mémoire de master en Lettres Classiques, sous la direction de Dominique Briquel, Paris, Université Paris-Sorbonne, 2013.

21 M. Morandi, Prosopographia etrusca, vol. I, Rome, 2004, p. 74-75.

22 M. Pallottino, "Oriundi forestieri nella onomastica e nella società etrusca », in M. G. Marzi Costagli (a cura di), Studi di Antichità in onore di G. Maetzke, Rome, I984, p. 40I-405 : parmi les gentilices de dérivation ethnique, ceux à suffixe - at/ $\theta e$ se réfèrent à des territoires non-étrusques. Leur diffusion est récente.

23 M. Morandi, Prosopographia etrusca, vol. I, Rome, 2004, p. 456-457. 
des sépultures, la présence du matronyme dans des inscriptions honorifiques, le fait qu'il existait un système d'alliances matrimoniales se profilant derrière cet élément onomastique, le fait que le matronyme met l'accent sur l'origine familiale de l'épouse) on voit se dessiner une idéologie qui mêle vie privée et vie sociale et fait du matronyme un signe potentiel de prestige lié à l'importance socio-politique du mariage. Puisque les femmes mariées gardaient le nom de leur père, il est tout à fait possible que dans certains cas, le matronyme soit un élément de prestige parce qu'il souligne l'ascendance maternelle du défunt. Autrement dit, l’identité de son grand-père maternel.

Cela dit, il faut se souvenir que le matronyme est avant tout un élément onomastique, autrement dit, un moyen d'identifier une personne. Pour ne pas le surinterpréter, il faut prendre en considération son côté pratique. Prenons le troisième matronyme de la Tombe de Boucliers. Il fait partie d'une épitaphe simple,

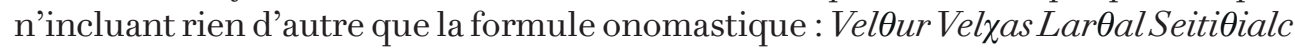
clan $^{\mathbf{2 4}}$ 'Velthur Velchas fils de Larth et de Seitithi'. Il y a deux façons d'expliquer pourquoi l'on a décidé d'insérer un matronyme ici. Velia Seitihi était l'épouse du personnage central de l'hypogée, son fondateur Larth I. L’emploi du matronyme peut être donc une forme d'hommage à Velia et à sa famille. Mais il y aussi une autre explication possible, de nature pratique : comme nous l'avons mentionné, la Tombe des Boucliers se compose de plusieurs chambres sépulcrales. La chambre B est la plus ancienne, la C et la D sont plus récentes. Sur les parois de la chambre sépulcrale principale, la chambre $B$, sont peints quatorze boucliers, et quatorze sont les membres de la famille représentés sur la fresque de la chambre A. Il est naturel d'imaginer qu'il y a une correspondance entre ces deux chiffres et que les quatorze personnes représentées sur la fresque étaient destinées à la chambre $\mathrm{B}$, vraisemblablement au moment où l'hypogée a été construit. Or, le matronyme dont nous sommes en train de discuter appartient à Velthur II, fils du fondateur Larth I. Velthur II apparaît sur la fresque, dans la scène de la procession plus précisément, à côté de son père, de sa mère Velia Seitithi et de sa soeur Velchai. Pourtant, il n'est pas dans la chambre B. Son nom apparaît sur la paroi adjacente à la chambre D, indiquant qu'il y est déposé. À sa place, dans la chambre B, est indiquée la présence de son fils Larth II, qui pourtant n'est pas représenté sur la fresque puisqu'il n'était probablement pas né à l'époque. Notons que, comme l'épitaphe de Velthur II, l'épitaphe de Larth II n'est pas peinte à l'intérieur de la chambre sépulcrale mais sur la paroi de la chambre A adjacente à la chambre sépulcrale. Mais les épitaphes pariétales des autres personnes déposées dans la chambre B sont peintes à l'intérieur de la chambre. Les épitaphes pariétales de cet

24 Les inscriptions dont la lecture et l'interprétation ne posent pas de difficultés sont, par commodité, transcrites sans signes diacritiques, avec des majuscules pour les noms propres. 
hypogée appartiennent donc à deux catégories : celles qui ont été peintes à l'intérieur des chambres sépulcrales (catégorie à laquelle appartiennent la majorité des épitaphes de la chambre B) et celles qui ont été peintes sur les parois adjacentes (catégories à laquelle appartiennent les épitaphes de Velthur II et Larth II). Ces choix relèvent de logiques sépulcrales ou décoratives un peu différentes, séparées peut-être par un laps de temps. De l'ensemble de ces observations nous pouvons déduire le scénario suivant : Velthur II était destiné à la chambre B avec les autres membres de la famille représentés sur la fresque, mais quand le moment est arrivé de l'enterrer, et que l'hypogée avait déjà été utilisé pendant un moment, pour une raison ou pour une autre, les personnes en charge de l'hypogée ont modifié les plans initiaux. Elles ont enterré Velthur II dans la chambre D et son fils Larth II dans la chambre B. Elles ont interverti le père et le fils. C'est peut-être pour cette raison que la formule onomastique de Velthur II a été indiquée avec autant de précision : avec trois Velthur présents dans l'hypogée et ce changement d'emplacement, le matronyme rendait parfaitement clair où se trouvait le fils du fondateur.

\section{Conclusion}

Pour ne pas surinterpréter la signification sociale du matronyme, il est important de ne pas oublier un constat préliminaire : comme l'a suggéré Benelli dans son analyse de la Table de Cortone, le matronyme pouvait être parfois un simple moyen d'identifier un individu. Cela dit, il ressort de notre analyse que le matronyme avait bien une valeur intrinsèque et qu'il est révélateur de l'importance que l'on accordait à la matrone étrusque. La valeur du matronyme, toutefois, n'est pas à chercher du côté du féminisme dans le sens moderne du terme : le matronyme n'est pas un élément onomastique équivalent au patronyme et il met beaucoup plus l'accent sur les origines familiales de la mère que sur la mère ellemême. La présence du matronyme dans de grandes inscriptions au ton soutenu et les politiques matrimoniales de haut niveau qui se profilent derrière cet élément onomastique nous renvoient à l'idéologie aristocratique que les hypogées souterrains, domaine privilégié du matronyme, essayaient d'expliquer.

Une contextualisation historique peut être profitable: au cours du Ve siècle av. J.-C., la société tarquinienne ne traverse une période de transition politique de la monarchie à l'oligarchie. Ce changement, ainsi que l'intense urbanisation $\mathrm{du} \mathrm{IV}^{\mathrm{e}}$ siècle qui a favorisé le développement de la classe moyenne, mènent à une crise de l'aristocratie ${ }^{25}$. Or, les matronymes que nous avons étudiés datent tous d'entre le $\mathrm{IV}^{\mathrm{e}}$ et le II ${ }^{\mathrm{e}}$ siècle av. J.-C. C'est précisément au IV siècle que l'on voit

25 Archéologiquement, ces événements sont attestés par la diminution des hypogées aristocratiques et par une architecture funéraire plus uniforme et moins monumentale, 
une résurgence de l'orgueil gentilice. Les hypogées de cette époque ont en effet une aura aristocratique bien visible : les portraits des défunts et de leurs ancêtres (peints sur paroi ou sculptés sur sarcophage), les nombreuses inscriptions relatant généalogies et carrières, un culte des ancêtres dont il existe quelques indices ${ }^{26}$, ainsi que les banquets et les processions de magistrats, récurrents en iconographie, valorisent le clan, ses privilèges et l'unité de la classe aristocratique. La femme a un rôle bien spécifique dans ce système idéologique, et c'est ce rôle qui est mis en valeur dans les hypogées : l'épouse est un moyen d'échange à l'intérieur d'un cercle qui se veut solidaire et clos, la mère est le lien entre un individu et son ascendance du côté maternel (le grand-père maternel, l'arrière-grand-père maternel, etc.).

Cela explique pourquoi le matronyme est si peu présent sur support externe. Il semble presque toujours trouver sa place dans les hypogées. Le patronyme est aussi plus fréquent dans les hypogées, où l'espace disponible pour écrire était plus abondant, mais sur support externe, lorsque l'espace épigraphique était limité, il a toujours priorité sur le matronyme. Le nom de la mère ne pouvait pas avoir la même importance concrète dans la vie d'un individu que celui du père : en effet, dans une société patriarcale (et il n'y pas de raison de croire que la société étrusque ne rentrait pas dans cette catégorie), la figure paternelle est au fondement de l'identité. L'analyse du corpus épigraphique de Tarquinia suggère que le matronyme était un élément onomastique utile et prestigieux, pas une information que l'on percevait comme importante dans n'importe quel contexte.

\section{Lavinia Magnani}

Master 2 Lettres Classiques,

Université Paris-Sorbonne

École Normale Supérieure

24.5 Canal Point - 22 West Tollcross

Edinburgh - EH3 9QW

lgt.magnani@cantab.net

voir S. SteIngräвer, Etruskische Wandmalerei. Von der geometrischen Periode bis zum Hellenismus, Munich, 2006, p. I3I.

26 L'on peut supposer des références à un culte des ancêtres dans l'image de l'héroïsation de Velthur Velchas (Tombe des Boucliers). 
